\title{
Retinal Signal Transmission in Duchenne Muscular Dystrophy: Evidence for Dysfunction in the Photoreceptor/Depolarizing Bipolar Cell Pathway
}

\author{
Kathleen M. Fitzgerald, ${ }^{*}$ Gerhard W. Cibis, ${ }^{\star \dagger}$ Steven A. Giambrone, ${ }^{*}$ and David J. Harris ${ }^{8}$ \\ *Vision Sciences Laboratory, Sections of ${ }^{\ddagger}$ Ophthalmology and ${ }^{\S}$ Genetics, The Children's Mercy Hospital \\ and ${ }^{\ddagger}$ The Eye Foundation of Kansas City, University of Missouri, Kansas City, Missouri 64108
}

\begin{abstract}
There have been reports of abnormal retinal neurotransmission determined by electroretinography in boys with Duchenne and Becker muscular dystrophy. Dystrophin may play a role in transmitting signals between photoreceptors and the excitatory synapse of the $\mathrm{ON}$-bipolar cell. These electroretinographic changes appeared to be limited to the rod $\mathrm{ON}$-pathway but we felt there was also similar abnormality in the cone ON-pathway. We used long-duration stimuli to separate ON (depolarizing bipolar cell) and OFF (hyperpolarizing bipolar cell) contributions to the cone-dominated ERG to better understand how the retina functions in boys with Duchenne muscular dystrophy. We recorded the electroretinograms of 11 boys with Duchenne muscular dystrophy and found abnormal signal transmission at the level of the photoreceptor and $\mathrm{ON}$-bipolar cell in both the rod and cone generated responses. The OFF-bipolar cell that responds to the offset of the stimulus continues to function normally. The results support our hypothesis that retinal dystrophin plays a role in receptor function or controlling ion channels at the level of the photoreceptor and depolarizing bipolar cell. (J. Clin. Invest. 1994. 93:2425-2430.) Key words: sensory neurons • electroretinography • dystrophin • retina $\cdot$ glutamate receptors
\end{abstract}

\section{Introduction}

Photoreceptor cells (rods and cones) continuously release the neurotransmitter L-glutamate in the dark. The synaptic release of L-glutamate is inhibited under light stimulation when the cells hyperpolarize (1). The reduction in neurotransmitter release causes the bipolar cells (second-order neurons) to either depolarize or hyperpolarize dependent upon their unique receptors (2). Depolarizing bipolar cells $(\mathrm{DBCs})^{1}$ drive ganglion cells that fire at the onset of light whereas hyperpolarizing bipo-

This work was presented in part at the Annual meeting of the Association for Research in Vision and Ophthalmology, Sarasota, FL, 2-7 May, 1993.

Address correspondence to Kathleen Fitzgerald, R. EP T., Ph.D., Vision Sciences Laboratory, The Children's Mercy Hospital, 2401 Gillham Road, Kansas City, MO 64108-9898.

Received for publication 29 October 1993 and in revised form 18 January 1994.

1. Abbreviations used in this paper: CSNB, congenital stationary night blindness; DBC, depolarizing bipolar cell; DMD, Duchenne muscular dystrophy; ERG, electroretinography; HBC, hyperpolarizing bipolar cell.

J. Clin. Invest.

(C) The American Society for Clinical Investigation, Inc.

$0021-9738 / 94 / 06 / 2425 / 06 \$ 2.00$

Volume 93, June 1994, 2425-2430 lar cells (HBCs) drive ganglion cells that fire at the offset of light. DBCs and HBCs are also referred to as ON- and OFF-bipolar cells, respectively. Therefore, organization at the level of the retina begins for two parallel pathways: the ON-pathway, which responds to an increase in brightness and the OFF-pathway, which responds to a decrease in brightness (3).

Cones may synapse to either depolarizing ON- or hyperpolarizing OFF-bipolar cells. The cone ON-bipolar cell makes a direct synapse with $\mathrm{ON}$-ganglion cells, and, similarly, the cone OFF-bipolar cell synapses with an OFF-ganglion cell providing direct but distinctly different neural pathways to the visual cortex (4). Conversely, rods synapse to only one bipolar cell type, a depolarizing ON-bipolar cell (5). The output of the rod bipolar cell is not to a ganglion cell, instead, rod signals reach ganglion cells through an intermediate circuit: amacrine cells. The AII amacrine cell receives input from the rod bipolar cell and makes an electrical synapse on the cone ON-bipolar terminal causing it and the ganglion cell it contacts to depolarize (2). Using the inhibitory transmitter glycine, the AII amacrine cell hyperpolarizes the terminals of cone OFF-bipolars and the ganglion cells they contact. Therefore, while cones have a direct bipolar cell pathway to ganglion cells, the rods must use the cone bipolar cell pathway through the AII amacrine cell to send messages to ganglion cells.

There have been recent reports of abnormal retinal transmission in boys with Duchenne muscular dystrophy (DMD), a fatal neuromuscular disease that is the result of mutations in the dystrophin gene (6-8). Dystrophin is absent or nearly absent in DMD and it is possible that dystrophin plays a role in normal retinal function. The studies of retinal function have been done using electroretinography (ERG); a recording of the summed electrical signal produced by the retina when stimulated with a flash of light. The dark-adapted ERGs, recorded under scotopic testing conditions, have shown normal $a$-waves (a response of negative polarity generated by the photoreceptors) but reduced amplitude rod-isolated $b$-waves (a response of positive polarity originating primarily from the ON-bipolar cells) (9). This type of ERG abnormality with profound $b$-wave suppression is commonly associated with night blindness, (10) however, there have not been reports of night blindness or any other visual anomalies in boys with DMD, and dark-adaptometry studies have been normal (7).

The cone-mediated ERG recorded under photopic testing conditions has been reported as normal in DMD subjects (7, 11 ) or slightly reduced in amplitude, (8) however, we have observed the absence or attenuation of a small wavelet on the ascending $b$-wave of this response in our DMD subjects. Two small wavelets (oscillatory potentials $\mathrm{O} 1, \mathrm{O} 2$ ) are routinely seen on the ascending $b$-wave of the photopic ERG in our normal subjects but the second oscillatory potential, $\mathrm{O} 2$, was consistently absent in the DMD photopic ERG. Other evidence that pointed to a dysfunction at the level of the cone and its DBC was an unusual sawtooth-shaped waveform in the DMD 
$30 \mathrm{~Hz}$ flicker response. These clinical ERGs are recorded to short-duration stimuli of $.1 \mathrm{~ms}$ and such brief flashes of light do not separate cone DBC and HBC contributions; the clinical photopic ERG is a mixture of both ON- and OFF-responses. Long-duration stimuli are needed to isolate cone DBC and $\mathrm{HBC}$ activity in the retina.

Long-duration photopic ERGs were used by other investigators to study subjects with retinal ON-pathway disorders such as CSNB (12-14) and paraneoplastic night blindness (15). The authors found absent or severely reduced rod and cone DBC responses that suggested these disorders were specific to ON-pathways. The clinical ERG showed reduced amplitude or absent rod-dominated $b$-waves with an abnormal cone response; both $\mathrm{O} 1$ and $\mathrm{O} 2$ oscillatory potentials were missing. While the ERGs in both CSNB and paraneoplastic night blindness are similar to what we have described in our DMD subjects, there were significant exceptions that, in our opinion, distinguished the DMD ERG as a clinical entity distinct from CSNB and other negative ERGs: the photopic O1 oscillatory potential was intact, the photopic $\mathrm{O} 2$ oscillatory potential was attenuated or absent and sawtooth-shaped waveform in the $\mathbf{3 0}$ $\mathrm{Hz}$ flicker response. We questioned whether the abnormal ERG in DMD was limited to just the rod pathway as suggested by the absence of rod-dominated $b$-waves. The purpose of this study was to use long-duration stimuli to isolate DBC and HBC contributions to the photopic ERG in order to determine the source of the apparent ERG abnormalities. Here we show functional evidence that would support the hypothesis that retinal dystrophin may localize to the postsynaptic membrane of the DBCs.

\section{Methods}

Subjects. The subjects were recruited from The Children's Mercy Hospital Departments of Ophthalmology and Genetics after the tenets of the Declaration of Helsinki and Internal Review Board approval. Subjects were 11 male children between the ages of $2 \mathrm{yr}, 4 \mathrm{mo}$, and $21 \mathrm{yr}$ (mean; $9 \mathrm{yr}$ ) who met the standard criteria for DMD: muscle weakness, elevated CPK, a positive family history when present, or a consistent muscle biopsy. All subjects had Southern blot analysis of genomic DNA and $75 \%$ had demonstrable deletions in the DMD gene. The remaining $25 \%$ had non-detectable deletions, and the diagnosis of DMD was based on the clinical presentation and muscle biopsies. A complete eye examination was performed before the ERG.

$E R G$. All subjects underwent a clinical ERG to short-duration stimuli of varying wavelengths before the long-duration photopic ERG. The clinical ERGs were recorded following published guidelines (16). Methods of the clinical ERGs are described in a previous paper (6).

After the clinical ERG, long-duration photopic ERGs were recorded. After $10 \mathrm{~min}$ of light adaptation to a rod-desensitizing background of $20 \mathrm{ft}-\mathrm{L}$, a $2.2-\log \mathrm{cd}-\mathrm{s} / \mathrm{m} 2$ stimulus (Dolan-Jenner Industries, Inc., Woburn, MA) was delivered through a 3-mm fiberoptic cable (Dolan-Jenner Industries, Inc. Woburn, MA) which was presented on a 30-ft-L Ganzfeld background (Nicolet Biomedical Instrument Corp., Madison, WI). Flash and background luminance were calibrated with a photometer (model 350, United Detector Technology, Hawthorne, CA). A Uniblitz shutter (Vincent Associates, Rochester, NY) was used to control the duration of the stimulus. ERGs were recorded to stimulus durations of $100,80,60,40,20,10$, and $.1 \mathrm{~ms}$ to visualize the OFF-response merging with the ON-response. The ERG was recorded with a monopolar "jet" contact lens electrode (Universo SA, La Chaux-De-Fonds, Switzerland), referenced to the patients ipsilateral mastoid with a forehead ground. Recordings were performed with a Nicolet CA-1000 signal averaging system (Nicolet Biomedical Instru- ment Corp., Madison, WI). Ten repetitions were averaged using a $1-1,500 \mathrm{~Hz}$ ( $-3 \mathrm{~dB}$ points) bandpass in a $200 \mathrm{~ms}$ window. Results were stored on a floppy disk for later analysis. The $a$-wave amplitude was measured from the baseline to the trough of the $a$-wave. ON-response amplitude was measured from the trough of the $a$-wave to the peak of the $b$-wave. The $b / a$ amplitude ratio was obtained by dividing $b$-wave amplitude by $a$-wave amplitude.

\section{Results}

The eye examinations in all eleven subjects were normal with no abnormalities of the cornea, anterior chamber, iris, lens, vitreous, optic disk, retinal vessels, macula, or peripheral retina. Eye alignment was normal with no incidence of strabismus. There were no significant refractive errors. The subjects had no visual complaints. Their parents did not observe behavior associated with night blindness.

The clinical short-duration $(.1 \mathrm{~ms})$ ERG to both scotopic and photopic conditions is illustrated in Fig. 1. Representative normal and CSNB ERGs are shown for comparison to the DMD ERG. The normal subject shows a positive $b$-wave rise to very dim blue stimuli. This response is rod dominated, and, only when the intensity increases, do cone contributions become apparent. The negative polarity $a$-wave generated from both rod and cone photoreceptors is best seen at higher intensity white light. The $a$-wave is followed by the positive polarity $b$-wave that rises above the baseline yielding $b$-wave amplitudes that are approximately twice the amplitude of the $a$-wave. The scotopic oscillatory potential is filtered to remove slower frequencies giving a better view of the faster frequency components. After light-adaptation, cone dominated photopic responses are recorded in the presence of steady background light. To a short-duration $(.1 \mathrm{~ms})$ flash, a shallow $a$-wave of negative polarity is followed by a positive polarity $b$-wave of approximately four times the amplitude of the $a$-wave. Two small oscillatory potentials $(\mathrm{O} 1, \mathrm{O} 2)$ are seen on the ascending limb of the $b$-wave. The response to a $30 \mathrm{~Hz}$ flickering light is sinusoidal in waveform.

The representative CSNB ERG is typical for this disorder with no $b$-wave to scotopic stimuli. The response to a bright white flash is often termed "negative" because of the lack of positive-polarity $b$-wave rise. Scotopic oscillatory potentials are attenuated and of particular interest, the photopic oscillatory potentials, $\mathrm{O} 1$ and $\mathrm{O} 2$, are missing. The response to $30 \mathrm{~Hz}$ flicker is sawtooth in waveform.

The DMD ERG shares scotopic similarities to CSNB such as attenuated $b$-waves, a negative response to bright white stimuli and attenuated oscillatory potentials but the photopic response is unique because the $\mathrm{O} 1$ oscillatory potential is preserved and the $\mathrm{O} 2$ oscillatory potential is attenuated to absent. The $30 \mathrm{~Hz}$ flicker response is also sawtooth in its waveform but less so than CSNB.

Photopic ON- and OFF-responses to long-duration stimuli are illustrated in Fig. 2. In normal subjects, the negative-polarity $a$-wave is followed by the positive-polarity $b$-wave (ON-response) that rises above the stimulus baseline. This response occurs at the same place in time to all stimulus durations for the obvious reason that the onset of light occurs at the same time. The response to light offset (OFF-response) is seen shifting to the left as stimulus duration decreases. At shorter intensities, ON- and OFF-responses merge; both make contributions to the morphology of the short-duration photopic ERG and 


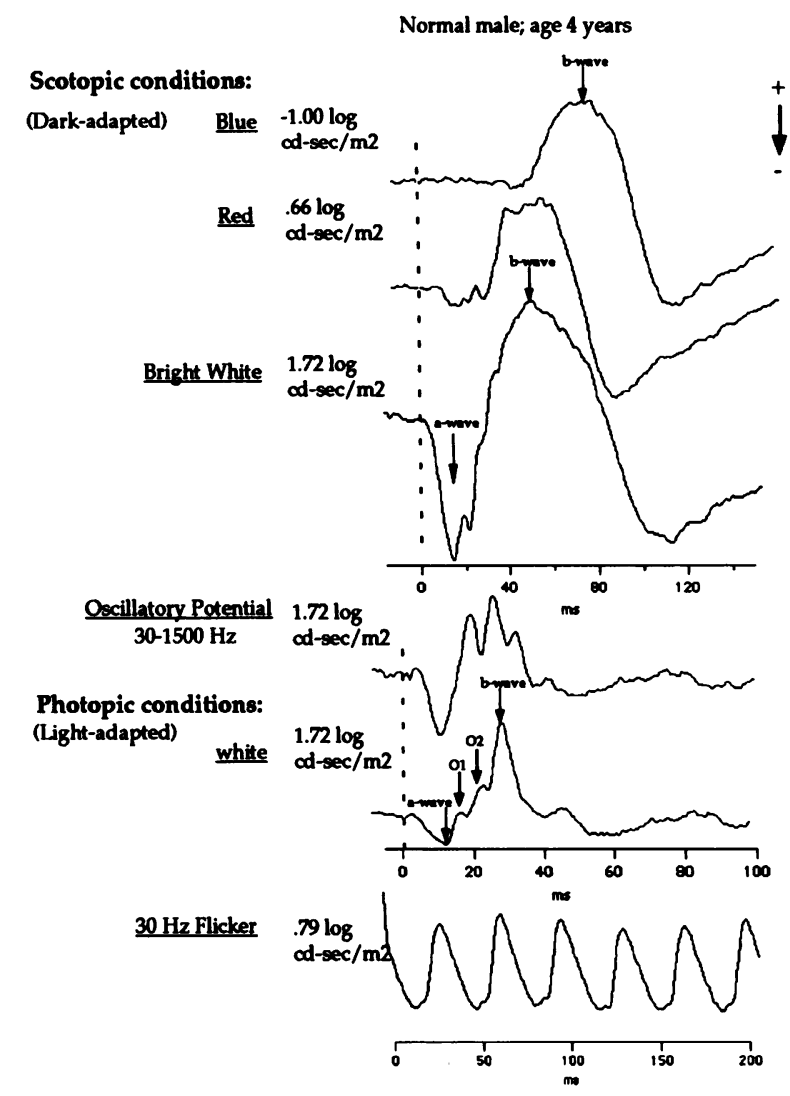

DMD, age 4 years

CSNB, age 7 years
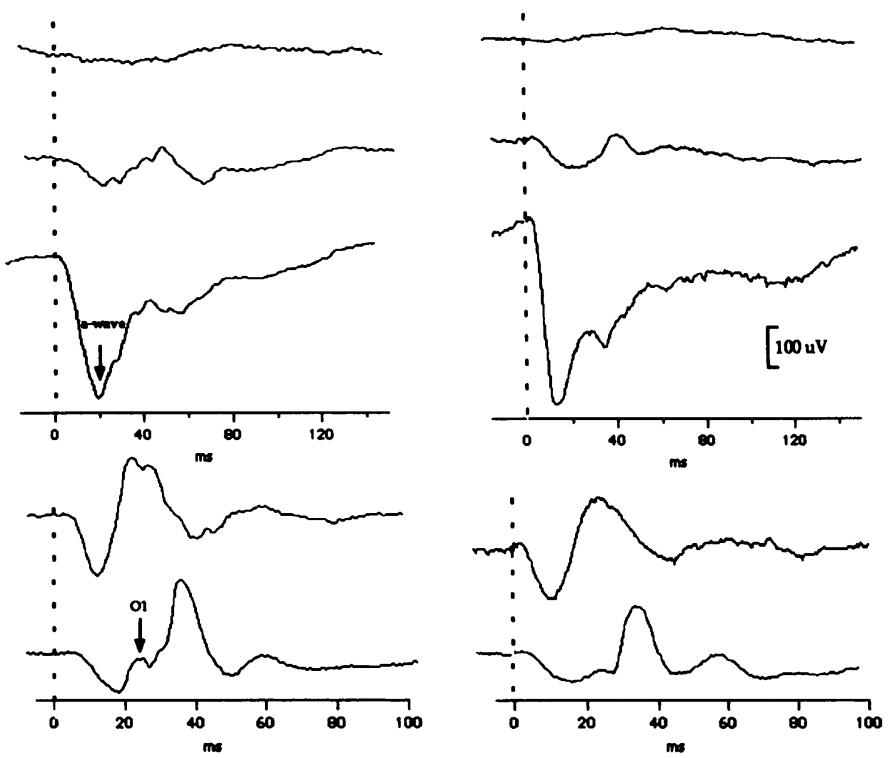

Figure 1. Clinical scotopic and photopic ERGs from representative normal, DMD and CSNB subjects. (DMD) Note the absence of a rod-mediated $b$-wave to blue, red, and white stimuli under scotopic testing conditions. Only cone oscillations can be seen in the response to the red stimulus. Response to a bright white flash shows only the photoreceptor generated $a$-wave. Under photopic testing conditions, the second oscillatory potential $(\mathrm{O} 2)$ is missing. The response to a $30 \mathrm{~Hz}$ flickering light shows a steeper leading edge followed by a less abrupt descending slope. (CSNB) Scotopic responses are similar to DMD with no rod contribution and a negative ERG to a bright white stimulus. Under photopic testing conditions, both $\mathrm{O} 1$ and $\mathrm{O} 2$ oscillatory potentials are missing. This has been identified as loss of the cone ON-response.

\section{Normal}

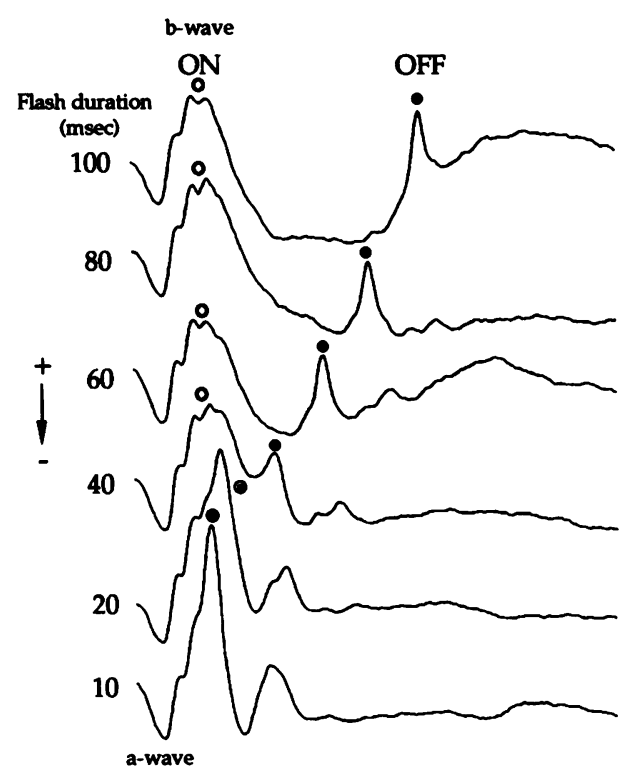

DMD

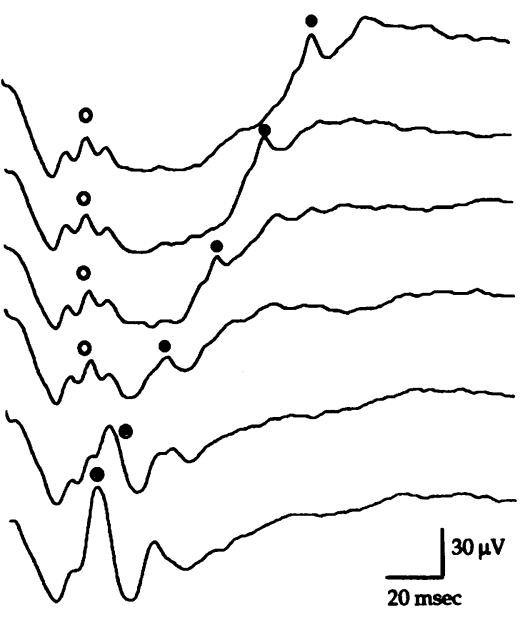

CSNB

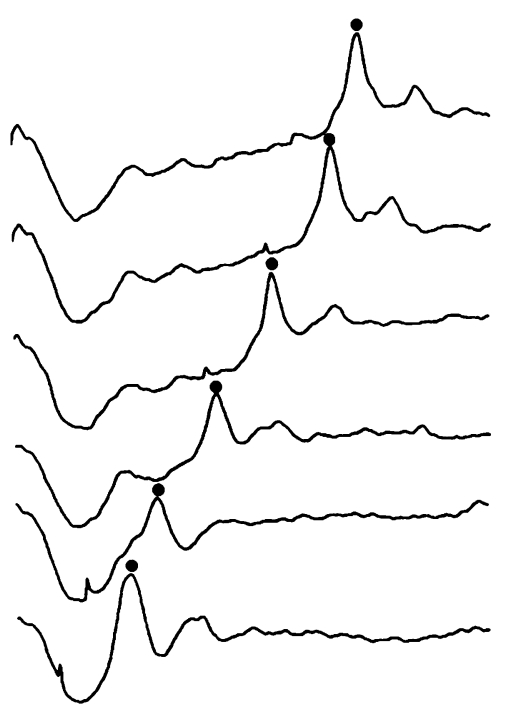

Figure 2. Long-duration cone-dominated photopic ERGs from representative normal, DMD and CSNB subjects. Open circles signify ON-responses, Solid circles signify OFF-responses, and the open circle with a dot is a mixture of both ON-and OFF-responses. The CSNB is shown for comparison to demonstrate an ON-pathway selective disorder; there is no response to stimulus onset. A small, decreased amplitude response to stimulus onset remains for DMD subjects but increased negativity of the $a$-wave and decreased amplitude ON-responses leads to abnormal ERG parameters to all stimulus durations. 
Table I. Mean and P-values for Long-duration (100-10 msec) and Short-duration (.1 msec) Photopic ERG

\begin{tabular}{|c|c|c|c|c|c|c|c|c|c|}
\hline \multirow{2}{*}{$\begin{array}{c}\text { Flash } \\
\text { duration }\end{array}$} & \multicolumn{3}{|c|}{ a-wave amplitude } & \multicolumn{3}{|c|}{ b-wave amplitude } & \multicolumn{3}{|c|}{ b/a amplitude ratio } \\
\hline & Normal & DMD & $P$-value* & Normal & DMD & $P$-value & Normal & DMD & $P$-value \\
\hline \multicolumn{10}{|l|}{ msec } \\
\hline 100 & 44.6 & 75.0 & .0024 & 86.4 & 43.1 & .0002 & 2.01 & .66 & $<.0001$ \\
\hline 80 & 45.6 & 62.7 & .0077 & 94.8 & 39.9 & $<.0001$ & 2.13 & .63 & $<.0001$ \\
\hline 60 & 49.8 & 64.2 & .0043 & 91.1 & 42.8 & $<.0001$ & 1.87 & .65 & $<.0001$ \\
\hline 40 & 45.8 & 64.0 & .0006 & 90.2 & 42.7 & $<.0001$ & 2.00 & .67 & $<.0001$ \\
\hline 20 & 48.9 & 69.9 & .0055 & 128.9 & 92.9 & .0281 & 2.75 & 1.36 & .0014 \\
\hline 10 & 50.9 & 72.1 & .0004 & 160.2 & 128.9 & .0713 & 3.23 & 1.75 & $<.0001$ \\
\hline .1 & 50.2 & 72.9 & .0028 & 230.2 & 159.6 & .0111 & 4.77 & 2.25 & $<.0001$ \\
\hline
\end{tabular}

${ }^{*} \operatorname{LOS}=.01$

oscillatory potentials are seen on the ascending limb of the $b$-wave.

The CSNB photopic ERG to long-duration stimuli is shown for comparison as a disorder affecting ON-pathways. The $a$-wave is of increased negative-polarity, the ON-response is highly attenuated to absent while the OFF-response is preserved. At short durations, the supposed $b$-wave is really the OFF-response and no oscillatory potentials can be seen on the ascending limb.

In contrast to CSNB, the DMD ERG retains some ON-response. The $a$-wave is of increased negative-polarity and a small positive response with oscillations can be seen following the $a$-wave. This ON-response is attenuated and does not rise above the baseline until the OFF-response contributes to the amplitude.

The ERGs were compared to age and gender-matched normal control subjects. Comparisons between groups were made using Student's $t$ test for unpaired measures (two-tailed). The means and P-values for ERG parameters to all stimulus durations are presented in Table $I$ and graphically represented in Figs. 3-5. Subjects with DMD show significantly increased $a$ wave amplitudes (Fig. 3) and low $b / a$ ratios (Fig. 4) to all flash

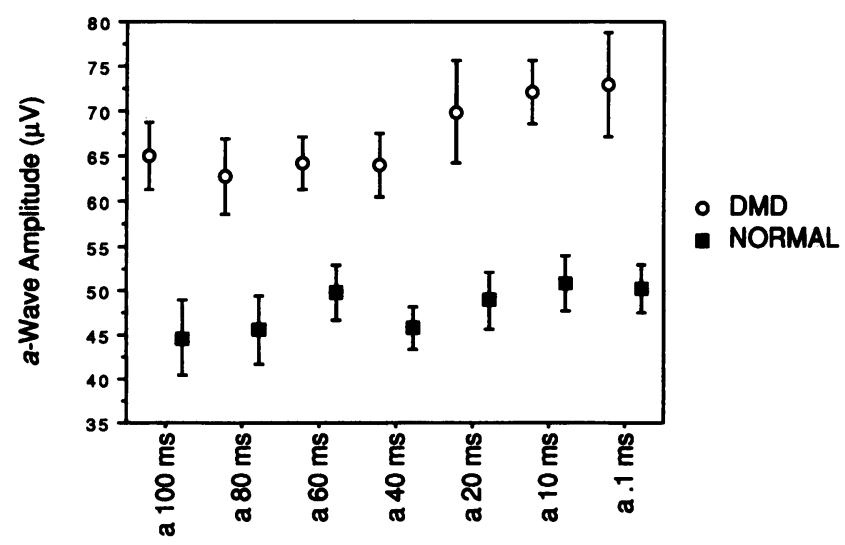

Figure 3. $a$-Wave amplitude of DMD subjects and normal controls to long- and short-duration stimuli. DMD $a$-wave amplitudes to long-duration stimuli are significantly increased in amplitude when compared to normal. The DMD $a$-wave continues to greater negativity because of the lack of a positive going $\mathrm{ON}$-response. Error bars represent \pm 1 SEM. durations. $b$-Wave amplitude is significantly decreased to all flash durations except 20,10, and .1 ms (Fig. 5). DMD photopic $a$-waves, like the scotopic $a$-wave, exhibits a strong negative trend resulting in increased $a$-wave amplitude. In addition, DMD subjects exhibit decreased amplitude of the photopic $\mathrm{ON}$-response ( $b$-wave) which represents the activity of the cone DBC. $b$-Wave amplitude varies significantly from normal except at 20,10, and .1 msec. At these shorter durations, the biphasic OFF-response merges with the ON-response increasing the amplitude of the $b$-wave; the shorter the duration, the steeper the $b$-wave. At $.1 \mathrm{~ms}$ (clinical photopic ERG) the ascending slope is very steep, resulting in what appears to be a normal $b$-wave. Traditionally, the $b$-wave is measured from the trough of the $a$-wave to the peak of the $b$-wave. If only $b$-wave values are considered, as in most clinical situations, the shortduration clinical photopic ERG would be considered normal. However, the ON-response is decreased in amplitude to all stimulus durations. This, coupled with increased negativity of the $a$-wave and summation of the OFF-response, adds to the

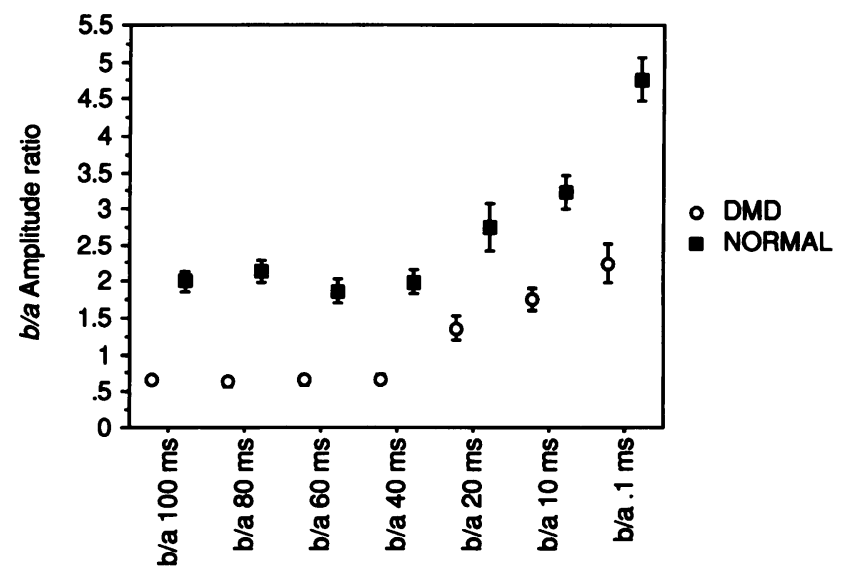

Figure 4. $b / a$ Amplitude ratios of DMD subjects and normal controls to long- and short-duration stimuli. In normal subjects the $b / a$ amplitude ratio is $>1.0$ to all stimulus durations. In contrast, DMD subjects show $b / a$ amplitude ratios that are $<1.0$ except at 20,10 , and $.1 \mathrm{~ms}$ when the amplitude of the OFF-response increases the amplitude of the $b$-wave which in turn gives higher $b$-wave values. Despite the addition of the OFF-response, the $b / a$ amplitude ratios are significantly lower than normal controls to all stimulus durations. Error bars represent \pm 1 SEM. 


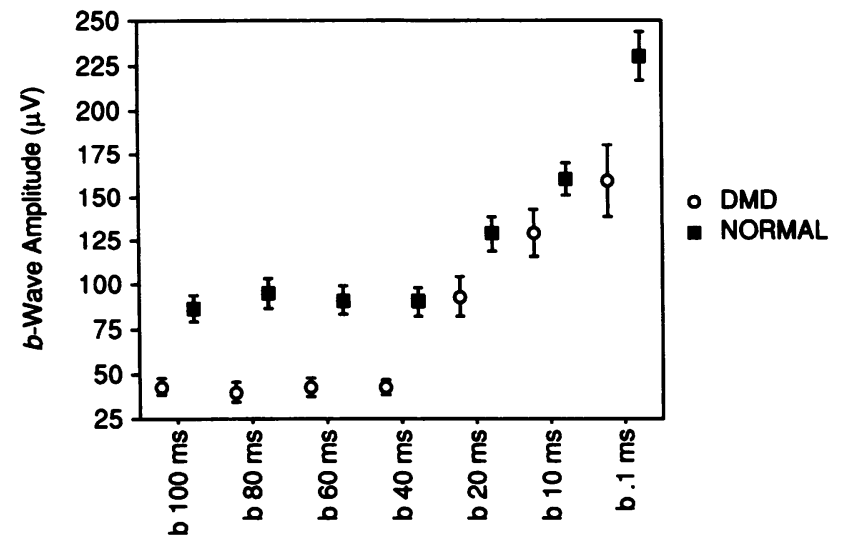

Figure 5. $b$-Wave amplitude of DMD subjects and normal controls to long- and short-duration stimuli. $b$-Wave amplitude is traditionally measured from the trough of the negative $a$-wave to the peak of the positive $b$-wave. $b$-Wave amplitude in DMD differs significantly from normal at the longer durations, but, as the OFF-response is added into the $b$-wave at shorter durations, the difference between groups is no longer significant. This gives the false impression that the clinical photopic ERG in DMD is normal, however, $a$-wave amplitude and $b / a$ amplitude ratios must be considered to fully analyze the photopic ERG. Error bars represent \pm 1 SEM.

value of the $b$-wave giving the false impression that $b$-wave amplitude is normal. This abnormality can only be detected in the clinical ERG by examining the $b / a$ amplitude ratios. In normal subjects, the $b / a$ amplitude ratio is $>1.0$ to all stimulus durations. In contrast to this, the $b / a$ amplitude ratio in DMD subjects is $<1.0$ to all stimulus durations except 20,10 , and .1 $\mathrm{ms}$, but, even with the contribution of the OFF-response, the $b / a$ ratio is significantly different at these shorter durations because of increased negativity of the $a$-wave and attenuation of the ON-response.

\section{Discussion}

The ERG in DMD is characterized by a negative response to a bright flash under scotopic testing conditions suggesting dysfunction at the level of the rod DBC. The clinical short-duration photopic ERG appears normal when only $b$-wave amplitude and implicit time are considered, however, the missing $\mathrm{O} 2$ oscillatory potential, increased negativity of the $a$-wave and decreased $b / a$ amplitude ratio points to cone $\mathrm{DBC}$ dysfunction as well. While the cone DBC response is highly attenuated, some function remains. This unique ERG could be the result of abnormal regulation of ion channels secondary to changes in function or structure of the DBC-specific receptor. Our ERG findings in $\mathrm{ON}$-pathway disorders have been consistent with the results of selectively blocking $\mathrm{DBC}$ and $\mathrm{HBC}$ contributions in the primate retina. These recent studies described below have added much to our understanding of the photopic ERG.

When ON-pathways (DBCs) are blocked with 2-amino-4phosphonobutyric acid (APB) the $a$-wave becomes more negative, the $b$-wave is suppressed, and the OFF-response is enhanced (17). This phenomenon is similar to the DMD and CSNB ERGs illustrated in Fig. 2. Subsequent blocking of HBCs, the OFF-pathway, with cis-2,3-piperdine dicarboxylic acid (PDA) reversed this effect and the $a$-wave becomes less negative, therefore, $\mathrm{HBCs}$ may make a contribution to the nega- tivity of the $a$-wave. When PDA and kynurenic acid were used alone to block the OFF-pathway, an early large amplitude light-evoked positive response was recorded that corresponded with the normal $b$-wave but was twice as large. It appears that the $\mathrm{DBC}$ ON-response contributes to the rising positive slope of the $b$-wave but the early persistent negativity of the HBC OFF-response truncates this large-amplitude positive wave. In a continuation of this work, Bush and Sieving (18) investigated the $30 \mathrm{~Hz}$ flicker in primates to determine whether the response might contain contributions from the inner retina. When ON-responses were blocked, they describe a major phase delay of the flicker that gives the waveform a sawtooth appearance, consistent with the $30 \mathrm{~Hz}$ flicker responses in DMD we report in this paper.

We believe the anomaly seen in the DMD photopic ERG and $30 \mathrm{~Hz}$ flicker can be explained by this "push-pull" mechanism between the ON- and OFF-pathways. The DBC ON-response has a fast velocity of positive polarity while the HBC OFF-response shows early negative polarity followed by a positive wave. The DMD photopic ERG shows increased negative $a$-wave amplitude and attenuation of the positive $\mathrm{O} 2$ oscillatory potential. If there is dysfunction of the cone $\mathrm{DBC}$ it would stand to reason that the DBC contribution would be dominated by the more negative $\mathrm{HBC}$ component leading to increased negativity and suppression of the leading edge of the $b$-wave, which possibly explains the missing $\mathrm{O} 2$ oscillatory potential.

Dystrophin is a large $(427 \mathrm{kD})$ protein with an amino acid sequence comparable to the spectrin family of membrane-associated cytoskeletal proteins (19). It exists in many isoforms, due to promoter selection and RNA splicing (20). Dystrophin localizes to the sarcolemma of skeletal, cardiac, and smooth muscle, and is thought to mechanically stabilize the plasma membrane, similar to erythrocyte spectrin (21). Another hypothesis is that dystrophin regulates the normal closure or voltage dependence of $\mathrm{Ca}^{+2}$ permeable membrane channels (22). Dystrophin has also been identified at cerebral cortical synapses but its role has yet to be elucidated (23). Recently, multiple isoforms of dystrophin were identified in the normal human retina (8).

It is possible that dystrophin isoforms play different roles in different tissues. Dystrophin binds to several glycoproteins in the sarcolemma membrane that surround muscle myofibrils. These associated proteins interact with dystrophin and may not exist in the same form in different tissues; indeed, it may prove that there will be dystrophin-glycoprotein complex models specific for separate tissues (24). We have not seen a correlation between the severity of the ERG abnormality and the severity of the muscle disease which suggest that the muscle and retinal products have different promoters and function (K. M. Fitzgerald, G. W. Cibis, A. H. Gettel, S. A. Giambrone, D. J. Harris, and R. A. White, manuscript in preparation).

If retinal dystrophin expression is required for normal function, our results give support to the idea that the dystrophinglycoprotein complex may play a role in signal transmission or formation of an ion channel. This is a reasonable hypothesis as a functionally related cytoskeletal protein, ankyrin, is associated with brain $\mathrm{Na}^{+}$channels (25). Monoclonal antibodies to an epitope of L-type (long-lasting) $\mathrm{Ca}^{+2}$ channels in hippocampal pyramidal neurons found the subcelluar localization to be identical to the location of dystrophin (26). Alterations in mature skeletal myocyte $\mathrm{Ca}^{+2}$ channel physiology and increased 
levels of intracellular $\mathrm{Ca}^{+2}$ concentrations have been found in boys with DMD (27). Mouse retinal bipolar cells have been found to have T-type (transient) $\mathrm{Ca}^{+2}$ channels (28). $\mathrm{Ca}^{+2}$ currents were found to be generated at both the perikaryon and at the axon terminal indicating that this current may participate in synaptic transmission. It is not known whether there are increased $\mathrm{Ca}^{+2}$ concentrations in the retina of DMD boys. Histology and immunohistochemical studies of DMD and normal human retina are needed to improve our understanding of retinal dystrophin.

We believe the abnormal ERG in DMD represents an ONpathway defect and is not a form of CSNB. Unlike CSNB, the cone $\mathrm{DBC} O \mathrm{ON}$-pathway retains some function. We suggest that the cone DBC response, while abnormal, maintains enough function to serve as a pathway for rods to transmit signals to ON-ganglion cells via AII amacrine cells, sparing DMD subjects from night blindness. We are currently investigating this hypothesis. The fact that this abnormal retinal function occurs in DMD, a well-studied population with no history of retinal disease and normal dark-adaptometry, suggests that dystrophin plays a role in normal retinal function. By using long-duration flashes to separate $\mathrm{DBC}$ and $\mathrm{HBC}$ activity we give functional evidence to support the hypothesis that retinal dystrophin may localize to depolarizing bipolar cells. This was suggested by Tamura et al. (29) and our results support that hypothesis. The results of this study give functional evidence to that role and favor localization of retinal dystrophin to the postsynaptic membrane of the DBC.

\section{Acknowledgment}

The authors gratefully acknowledge the technical expertise of Lori Moore, R. EEG/EP T., and thank Robert A. White, Ph.D., E. Eugenie Hartmann, Ph.D., and Vance Zemon, Ph.D. for critical review of the manuscript. This study was supported by a grant from the Katherine B. Richardson Associates.

\section{References}

1. Massey, S. C. 1982. Cell types using glutamate as a neurotransmitter in the vertebrate retina. In Progress in Retinal Research. N. N. Osborne and G. Chader, editors. Pergamon, Oxford, England. 399-425.

2. Kolb, H. 1991. The neural organization of the human retina. In Principles and Practice of Clinical Electrophysiology of Vision. J. R. Heckenlively and G. B. Arden, editors. Mosby Year Book, St. Louis, Missouri. 25-52.

3. Dowling, J. E. 1987. The retina: an approachable part of the brain. The Belknap Press of Harvard University Press, Cambridge, Massachusetts. 282 pp.

4. Schiller, P. H. 1982. Central connections of the retinal ON and OFF Pathways. Nature (Lond.). 197:580-583.

5. Wassle, H., M. Yamashita, U. Greferath, U. Grunert, and F. Muller. 1991 The rod bipolar cell of the mammalian retina. Visual Neurosci. 7:99-112.

6. Cibis, G. W., K. M. Fitzgerald, D. J. Harris, P. G. Rothberg, and M. Rupani. 1993. The effects of dystrophin gene mutations on the ERG in mice and humans. Invest. Ophthalmol. \& Visual Sci. 34:3646-3652.

7. DeBecker, I., J. Dooley, and F. Tremblay. 1993. Pathognomonic negative electroretinogram but not congenital stationary night blindness in Duchenne muscular dystrophy. Invest. Ophthalmol. \& Visual Sci. 34:1076. (Abstr.)

8. Pillers, D. A. M., D. E. Bulman, R. G. Weleber, D. A. Sigesmund, M. A. Musarella, B. R. Powell, W. H. Murphey, C. Westall, C. Panton, L. E. Becker, R. G. Worton, and P. N. Ray. 1993. Dystrophin expression in the human retina is required for normal function as defined by electroretinography. Nature Genet. 4:82-86.

9. Gurevich, L., and M. M. Slaughter. 1993. Comparisons of the waveforms of the ON bipolar neuron and the b-wave of the electroretinogram. Vision Res. 33:2431-2435.

10. Ripps, H. 1982. Night blindness revisited: from man to molecules. Invest. Ophthalmol. \& Visual Sci. 23:588-609.

11. Sigesmund, D. A., P. N. Ray, D. M. Pillers, R. G. Weleber, C. A. Westall, C. M. Panton, and M. A. Musarella. 1993. Duchenne muscular dystrophy (DMD); severity of rod dysfunction correlates with location of deletion in DMD gene. Invest. Ophthalmol. \& Visual Sci. 34:1463 (Abstr.).

12. Miyake, Y., K. Yagasaki, M. Horiguchi, and Y. Kawase. 1987. On- and off-responses in photopic electroretinogram in complete and incomplete types of congenital stationary night blindness. Jpn. J. Ophthalmol. 31:81-87.

13. Young, R. S. L. 1991. Low-frequency component of the photopic ERG in patients with X-linked congenital stationary night blindness. Clin. Vision Sci. 6:309-315.

14. Houchin, K. W., R. L. Purple, and J. D. Wirtschafter. 1991. X-Linked congenital stationary night blindness and depolarizing bipolar system dysfunction. Invest. Ophthalmol. \& Visual Sci. 32:1229 (Abstr.).

15. Alexander, K. R., G. A. Fishman, N. S. Peachey, A. L. Marchese, and M. O. M. Tso. 1992. 'On' response defect in paraneoplastic night blindness with cutaneous malignant melanoma. Invest. Ophthalmol. \& Visual Sci. 33:477-483.

16. Marmor, M. F., G. B. Arden, S. E. G. Nilsson, and E. Zrenner. 1989. Standard for clinical electroretinography. Arch. Ophthalmol. 107:816-819.

17. Sieving, P. A., K. Murayama, and F. Naarendorp. 1993. Push-pull mechanism of the primate photopic electroretinogram b-wave. In Ophthalmic \& Visual Optics/Noninvasive Assessment of the Visual System Technical Digest. Optical Society of America, Washington, DC. 318-320.

18. Bush, R. A., and P. A. Sieving. 1993. Monkey $30 \mathrm{~Hz}$ flicker ERG is generated partially by activity post-synaptic to cones. Invest. Ophthalmol. \& Visual Sci. 34:1273 (Abstr.).

19. Koenig, M., A. P. Monaco, and L. M. Kunkel. 1988. The complete sequence of dystrophin predicts a rod-shaped cytoskeletal protein. Cell. 53:219228.

20. Feener, C. A., M. Koenig, and L. M. Kunkel. 1989. Alternative splicing of human dystrophin mRNA generates isoforms at the carboxy terminus. Nature (Lond.). 338:509-511.

21. Kramarcy, N. R., and R. Sealock. 1990. Dystrophin as a focal adhesion protein. FEBS (Fed. Eur. Biochem. Soc.) Letters. 274:171-174.

22. Franco, A., and J. B. Lansman. 1990. Calcium entry through stretch-inactivated ion channels in mdx myotubes. Nature (Lond.). 344:670-673.

23. Lidov, H. G. W., T. J. Byers, S. C. Watkins, and L. M. Kunkel. 1990. Localization of dystrophin to postsynaptic regions of central nervous system cortical neurons. Nature (Lond.). 348:725-728.

24. Ervasti, J. M., and K. P. Campbell. 1993. Dystrophin and the membrane skeleton. Curr. Opin. Cell Biol. 5:82-87.

25. Srinivasan, Y., L. Elmer, J. Davis, V. Bennett, and K. Angelides. 1988. Ankyrin and spectrin associate with voltage-dependent sodium channels in brain. Nature (Lond.). 333:177-180.

26. Westenbroek, R. E., M. K. Ahlijanian, and W. A. Catterall. 1990. Clustering of L-type $\mathrm{Ca} 2+$ channels at the base of major dendrites in hippocampal pyramidal neurons. Nature (Lond.). 347:281-284.

27. Mehler, M. F., K. Z. Haas, J. A. Kessler, and P. K. Stanton. 1992. Enhanced sensitivity of hippocampal pyramidal neurons from mdx mice to hypoxia-induced loss of synaptic transmission. Proc. Natl. Acad. Sci. USA. 89:24612465.

28. Kaneko, A., L. H. Pinto, and M. Tachibana. 1989. Transient calcium current of retinal bipolar cells of the mouse. J. Physiol. 410:613-629.

29. Tamura, T., K. Yoshioka, Y. Jinno, N. Niikawa, and T. Miike. 1993. Dystrophin isoforms expressed in the mouse retina. J. Neurol. Sci. 115:214-218. 